\title{
A Tale of Two Nerves in Thyroid Surgery
}

\author{
Chintamani $^{1}$
}

Published online: 13 October 2017

(C) Association of Surgeons of India 2017

Surgery of thyroid gland is essentially about preservation of two nerves and two glands on either side and removal of the rest. The emphasis is thus on preservation, and a thyroid surgeon would patiently chase these four vital structures in each case of total thyroidectomy. Surgeons operating by clock have no business to be in the neck that has too many vital structures tightly packed together [1].

According to a published report in 2001, more than $50 \%$ litigations in endocrine surgery were related to thyroid surgery, and of these, more than $70 \%$ had to do with the recurrent laryngeal nerve (RLN) [2]. With a better understanding of the surgical anatomy of thyroid and its related surroundings, we now know that the other and often "forgotten" nerve, i.e., external branch of superior laryngeal nerve (EBSLN), is equally important if not more. EBSLN is also called the "nerve of Amelita Galli Curci" after a legendary opera singer. Her singing career ended due to damage to this nerve while undergoing surgery for goiter under local anesthesia. In earlier studies, it was often reported that the nerve could only be seen in less than $50 \%$ cases. However, with meticulous surgery and patience, it is possible to sight it in more than $80 \%$ cases as has been reported in many recent studies and also by the author $[1$, 3-5].

\section{Identifying EBSLN}

The nerve is usually found in the "space of Reeves" that opens up once the medial dissection is performed at the superior pole

Chintamani

drchintamani7@gmail.com

1 VMMC, Safdarjang Hospital, New Delhi, India
[1]. There are few basic principles while performing this maneuver. The ipsilateral lobe is given traction in a downward and outward direction thus opening up the sternothyroidlaryngeal triangle or "Jolls triangle" (Figs. 1 and 2). The triangle is bounded by sternothyroid muscle anteriorly and superior thyroid pole laterally.

Technique/trick to sight the nerve involves partial transection of sternothyroid muscle, identifying superior thyroid vessels that divide into anterior and posterior branches. These branches are ligated individually and as caudally as possible. Mass ligature (i.e., tying the artery and vein together can cause inadvertent damage to the nerve and there is also a risk of development of arterio-venous fistula).

\section{Classification of EBSLN Using Its Anatomical Relationship with the Pole (Cernea Classification) [5]}

Type-1: Most common presentation, i.e., 60-68\%. Nerve seen crossing the superior thyroid vessels $>1 \mathrm{~cm}$ or more above a horizontal plane passing through the upper border of superior thyroid pole.

Type-2a: Nerve crossing the vessel $<1 \mathrm{~cm}$ above the horizontal plane of the superior thyroid pole.

Type-2b: Nerve below the plane of superior thyroid pole (highest risk of damage).

Type-IV: The EBSLN runs dorsally to the superior thyroid pedicle.

The usual morbidity of damage to the EBSLN is loss of pitch, timber, and quality of voice that may jeopardize the professional life of singers, preachers, and of all those that use higher pitch notes of speech. Patients following surgery for thyroid must always undergo a quality of voice assessment and also indirect laryngoscopy as the damage may not always 


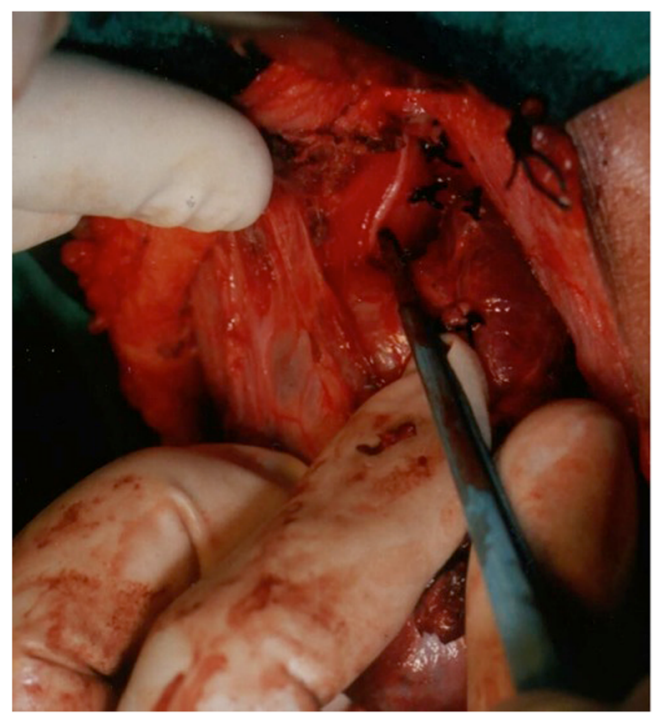

Fig. 1 The external branch of superior laryngeal nerve type 2a identified following the maneuver to open up the space of Reeves

be very apparent. In case of damage to this nerve, there are distinct features revealed on indirect laryngoscopy [vocal cord is shorter, hyperemic, and may be at a lower level, glottic chink may be oblique due to rotation of posterior commissure to paralyzed side].

\section{Recurrent Laryngeal Nerve}

This nerve has been studied extensively, being more closely linked with thyroid surgery, was first described by Galen in the second century. Unlike in the past when it was believed that "seen is damaged", the paradigm shifted long back to

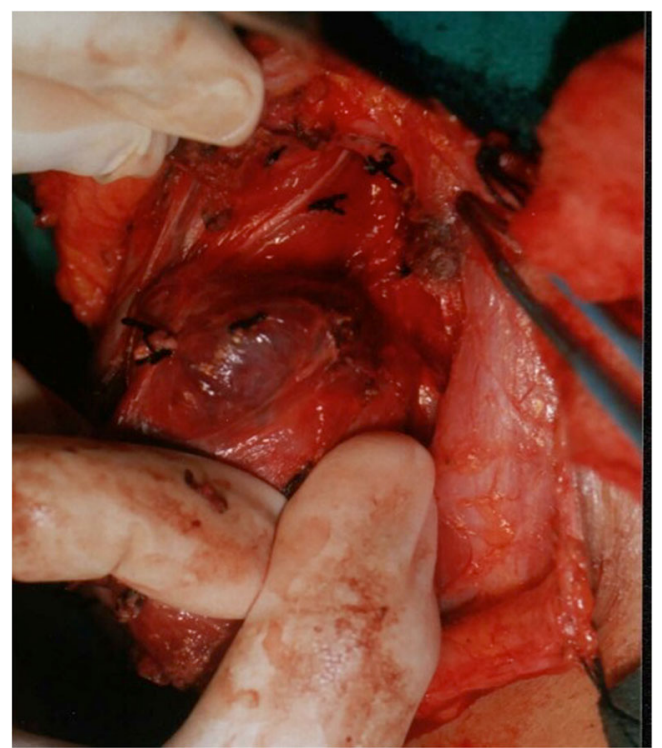

Fig. 2 The external branch of superior laryngeal nerve type 2a identified following the maneuver to open up the space of Reeves

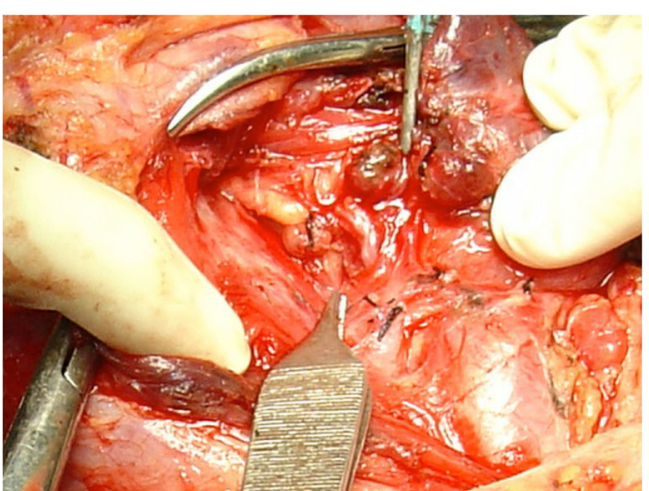

Fig. 3 The tubercle of Zuckerkandl: the RLN is seen along with parathyroid and sentinel fat of pad on lifting the tubercle. The tubercle along with the pyramidal lobe seen in the specimen

"not seen is damaged". (Prioleau 1933 "It is an axiom in Thyroid surgery that a RLN seen is injured".)

Billroth initially and Lahey (1933) later insisted that the RLN must be seen and preserved in all cases. The incidence of RLN injury varies in various studies but is somewhere between 0 and $13 \%$. The true incidence may be higher as a unilateral injury may get compensated by the opposite side and go unnoticed $[1,4,5]$.

\section{Identification and Preservation}

There are many approaches to identify the nerve but the three most commonly followed include, inferior, lateral, and superior approach. [The approach most often followed by the author is lateral or rarely superior]. Inferior approach is useful when one is dealing with a very large goiter or a recurrence. The approach needs to be tailored to the individual case and training of the surgeon.

Author would like to perform capsular dissection after handling the superior pole, and the nerve shows up in the natural course of dissection. One may not have to make a deliberate

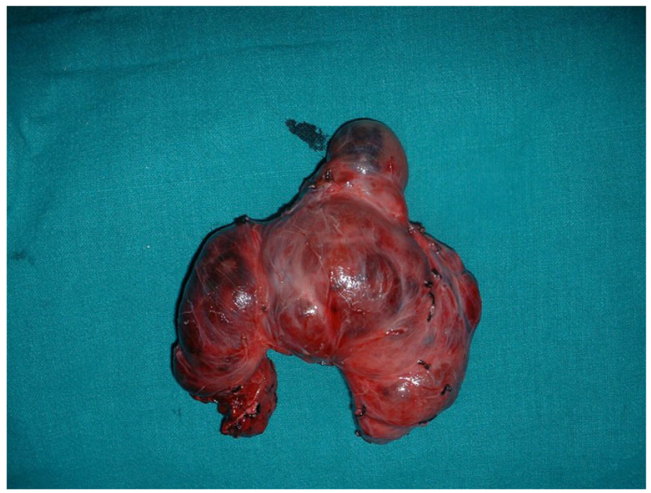

Fig. 4 The tubercle along with the pyramidal lobe seen in the specimen 


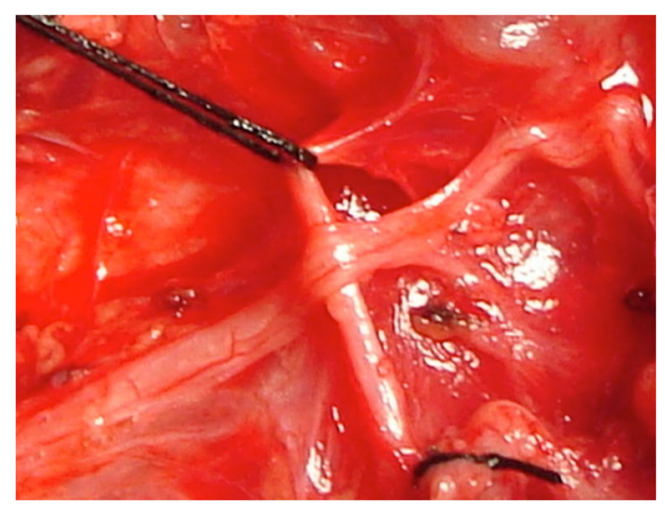

Fig. 5 The relationship of RLN with the inferior thyroid artery (on a sling). One can also appreciate the vasa nervosa on the RLN

attempt at searching unless the nerve does not show up in the routine progress of capsular dissection.

The nerve is identified as a white shiny structure with almost always a vein (vasa nervosa) running on its surface. The nerve should not be skeletonized as this may produce an ischemic damage (Fig. 5).

Lateral Approach After ligation of superior and inferior poles, the gland is retracted medially. The inferior parathyroid is carefully dissected away from the inferior pole and the nerve may be seen as a white shiny structure that feels like the string of a guitar.

Inferior Approach This approach involves identification of the nerve at thoracic inlet where it is often sighted in the RLN triangle [apex of triangle inferiorly formed by thoracic inlet, trachea medially and laterally the medial edge of retracted strap muscles while superiorly by the lower edge of the inferior pole]. The nerve exits as a single trunk here, and one has to take great care at not de-vascularizing the inferior parathyroid during dissection. The approach is suitable for large cervical goiters and a recurrent disease.

Superior Approach This approach involves identification of ligament of Berry_laryngeal point of entry and inferior cornu of thyroid cartilage is a useful landmark. This is useful for large and especially retrosternal goiters. One must take care to preserve the blood supply to superior parathyroid. This approach is technically more challenging.

RLN and Tubercle of Zuckerkandl (TBZ) This anomaly is indeed the friend of a surgeon and a reliable guide to the RLN [3]. The moment one lifts up the tubercle, RLN can be seen in most cases, as the nerve lies deep and medial to the tubercle (Figs. 3 and 4). This anomaly (site of fusion of median thyroid process and ultimo-branchial body) is seen in nearly $40-49 \%$ cases and can be graded as [grade 0:Un-recognizable, grade1: thickening of the lateral edge, grade-2: Smaller than $1 \mathrm{~cm}$, grade- 3: $>1 \mathrm{~cm}$ in size] [3].

RLN and Inferior Thyroid Artery Nerve lies deep to the artery and rarely may be going through the capsular branches of ITA (Fig. 5). One has to very carefully dissect the branches distal to the supply to parathyroid glands. The old dictum of ligating the inferior thyroid artery away from the gland and superior thyroid artery with the superior pole is now considered obsolete. One only ligates the capsular branches of this artery.

Non-recurrent laryngeal nerve is very rarely seen $(0.2-$ $0.4 \%$ ), is on the right side and is due to failed development of fourth aortic arch on right side.

Galen's Loop Rarely, the sensory branch of RLN may join the internal branch of superior laryngeal nerve to form Galen's loop.

Preservation of both these nerves along with parathyroid on either side is mandatory in thyroid surgery. Most likely sites for injury to the RLN that have been documented include the entry point at ligament of Berry or where the capsular branches of inferior thyroid artery are ligated and also the point where the nerve is entering the neck through thoracic inlet. The anatomy of the neck is by and large fixed, and one just needs to be patient and meticulous while performing thyroid surgery in order to preserve these vital structures.

\section{References}

1. Chintamani (2010) Editorial: "ten commandments" of safe and optimum thyroid surgery. Ten commandments. Indian J Surg 72(6): 421-426

2. Munch S, deKryger L (2001) Moral wounds: complicated complications [A Piece of My Mind]. JAMA 285(9):1131-1132

3. Chintamani (2013) "Friend or foe" of a thyroid surgeon? - the tubercle of Zuckerkandl. Indian J Surg 75(5):337-338

4. Aina EN, Hisham AN (2001) External laryngeal nerve in thyroid surgery: recognition and surgical implications. ANZ J Surg 71(4): 212-214

5. Mishra AK, Temadari H, Singh N, Mishra SK, Agarwal A (2007) The external laryngeal nerve in thyroid surgery: the "no more neglected' nerve. Indian J Med Sci 61(1):3-8 